\title{
Booked admissions as a replacement for waiting lists in the new NHS
}

\author{
Stephen Frankel, Joanna Coast, Tony Baker, Charles Collins
}

Admission arrangements for elective surgery often seem archaic and out of step with the public's experience of services outside the National Health Service. In a consumer oriented NHS it would clearly be preferable to offer most patients a mutually agreeable date for admission. We consider here the place of booked admission systems within the NHS and discuss surgeons' own experiences and preferences about booked admissions for elective surgery.

\section{Uncertainties}

The consideration of booking systems highlights a relatively unacknowledged aspect of waiting lists: uncertainty may be more important to patients than the length of time that they wait. Patients in the United States are said to wait longer on average for their elective surgery than those in Britain. ${ }^{1}$ These patients do not see waiting as a problem, however, because they know when their operations will take place.

Waiting lists effectively blur the boundaries of available provision by purporting to offer treatments when there may be little prospect of these treatments ever being delivered. This device is incompatible with the philosophy of the new NHS and is anyway politically unacceptable. The current reorganisation is informed by an interest in making explicit the limits to the care that may be offered, ${ }^{2}$ an interest which entails deciding somehow who is to be treated and who is not. ${ }^{3}$ If patients merit treatment then the capacity to provide it must be available and predictable. There should therefore be no reason not to offer patients admission dates when the decision to operate is made. Under these arrangements when patients cannot be accommodated in the hospital to which they have been referred the options may entail reconsidering the criteria for admission, reviewing resources (such as the number of operating lists or specialist staff available for elective surgery), or contracting additional facilities elsewhere. The limbo of the waiting list would no longer be available.

Two possible consequences of booking systems are particularly interesting. Firstly, surgeons who have introduced booking systems have reported a subsequent fall in the proportion of admissions contributed by emergencies. ${ }^{+5}$ Secondly, failed admissions may become less common with booking systems. ${ }^{56}$ These findings suggest that the order introduced by booking can spread more widely.

\section{Surgeon's views}

To be successful major policy changes in the organisation of elective surgery must build on existing practice and must accommodate the views and experience of those most directly implicated. For these reasons a questionnaire was sent to all surgeons in the South Western region to determine their practices and opinions about booking $(n=188$, response $140(74 \%))$. Twenty seven of the responding surgeons, including 15 of the 45 general surgeons, stated that they already offered an admission date to some patients needing non-urgent elective surgery during their clinic attendance. Surgeons who did not currently run booking systems were asked whether they would like to run one in the future: 36 said that they would prefer to book their inpatients and 43 their day cases. Many surgeons had given up a booking system in the past (49, including 24 general surgeons), but those unsatisfactory experiences did not mean they abandoned their interest in booked admissions. There was no relation between abandoning booking systems in the past and wishing to establish them in the future.

Only 31 consultants had no current or past experience of booking systems and no future interest in them. In other words, the majority $(109,78 \%)$ of surgeons either had operated a booking system, operated one now, or wanted to operate one in the future. Among general surgeons $39(86 \%)$ had some interest in them. Nevertheless, surgeons saw booking systems as being incompatible with the current organisation of hospital practice. Their responses to a number of open ended questions indicated the detailed issues that must be addressed for booking systems to succeed more widely. The conditions that might allow booking systems to succeed were clearly stated, and centred on protected facilities for elective surgery.

What was most pronounced in the responses was the level of frustration, particularly in response to the question of why consultants had abandoned booking arrangements. This is one example from many: "I prefer to run a booking system as I feel it improves the compliance of patients in attending for surgery. Unfortunately the volume and variability of emergency and urgent work has meant my booking system has collapsed with a large percentage of patients being cancelled, and I am therefore about to abandon it very reluctantly."

\section{Discussion}

The overall objective of the reformed National Health Service is to increase the efficiency with which health care is provided. One priority is to reduce waiting times and ideally to abolish waiting lists. These two broad objectives-efficiency and waiting list reduction-may in fact conflict.

Patients requiring elective procedures in the NHS must in general compete with urgent cases and those admitted as emergencies. The demands for urgent treatment fluctuate with a correspondingly inverse effect on the availability of beds and theatre time for elective surgery. The positive result of these arrangements is an efficient use of resources. But this efficiency is paid for by cancelled admissions ${ }^{7}$ and depends administratively on the amorphous queue known as a waiting list.

The uncertainty of generic waiting lists could be replaced by booking procedures analogous to those used by airlines. Such systems inevitably break down, however, when bookings are continually cancelled in deference to emergencies and urgent cases. Ending the continuing scandal of waiting lists must depend in part on successful booking systems. There success must depend in turn on beds and theatre time remaining available to those who are booked for admission. The scope for the efficient use of beds through what amounts to double booking is therefore diminished. 
In terms of throughput as measured by bed use waiting lists may be a more efficient way of admitting patients to hospital than a booked admissions system. But this ignores the costs of uncertainty borne by patients. These uncertainty costs may outweigh the costs of lost throughput and are anyway inconsistent with a consumer oriented health service where explicit decisions are to be made concerning the true intention to treat.

Surgeons are clearly interested in the extension of booking systems despite past failures and present frustration. They are also very explicit about the facilities required to make booked admissions practicable. These facilities are those that permit elective surgery to be protected from the demands of emergency and urgent cases. These predictable barriers have to be removed if any policy for introducing booking systems as a norm is to be successful.

We thank the consultant surgeons of the South Western region for their help in completing the questionnaires and Mrs Fiona Braddon for her help in analysing the responses.

1 Light D. Medical house arrest. Health Service fournal 1990; Nov 1:16+9. 2 National Health Service and Community Care Act 1990. London: HMSO, 1990. Dean M. Is your treatment economic, effective, efficient? Lancet 1991;337: $480-1$

Devin HB. Programmed elective surgery. In: Waiting for hospital treatmen. London: DHSS, 1980: 18-25. (Harrogate seminar reports).

Southam JA, Talbot RW. Planned surgical admissions in a district hospita $B M \mathcal{F} 1980$;ii:808-9.

6 Houghton PWJ, Brodribb AJM. Failure to attend for operation: a comparison

7 Frankel SJ, West RR, Farrow A Non-admission or non-initation? A case control study of failed admissions. BMY 1989;299:598-600.

\title{
How To Do It
}

\section{Edit a staff round}

\author{
Robert Winter
}

Examination of the $B M \mathcal{F}$ over its 151 years shows the staff round to be an established forum for clinical teaching, criticism, and advancing ideas. The weekly medical staff round at the then Postgraduate Medical School of London has been recorded in the $B M F$ at various times, the first clinicopathological conference appearing in 1959 and describing a 30 year old man with lupus nephritis and endocarditis. ${ }^{1}$ Publication of clinicopathological conferences in the $B M \mathcal{F}$ continued more or less regularly until 1960 , occasional contributions continuing up to 1978 .

Colleagues in North America have had the impressive weekly records of the case records of the Massachusetts General Hospital for 80 years since their foundation by Richard Cabot. ${ }^{2}$ The case records were first published in book form, but in 1915 Cabot's secretary began recording the discussion, including this along with the clinical and necropsy reports in the material circulated to a restricted list of physicians. Later still, these records were published in the Boston Medical and Surgical Fournal (now the New England Fournal of Medicine), marking the start of a series which later won world renown and which has continued without interruption to this day - a notable record. The records now form an important part of that journal; the section has a full time staff, and accounts of the records in the form of $35 \mathrm{~mm}$ colour slides and other audiovisual material are provided for educational purposes. Discussion of a single case in its wider context has a particular appeal, perhaps because for most the craft of medicine is learnt from treating individual patients and from sharing the experiences of others.

Most hospitals now hold a weekly staff round. This provides a formal occasion for clinical problems and their management to be discussed. Although the standard can vary, these presentations often provide an excellent overview of a clinical subject. This brief account describes how I have edited the medical staff round at Hammersmith for the $B M \mathcal{F}$ and gives guidelines to those planning to submit cases for publication.

Department of Medicine, Hammersmith Hospital, London W12 0HS

Robert Winter, MRCP, series editor, Hammersmith staff rounds

BMF 1991:303:1258-9 conditions such as thrombocytopenia in sarcoidosis, ${ }^{3}$ or of disorders whose incidence seems to be increasing or whose diagnosis may be easily overlooked-for example, systemic candidiasis. ${ }^{4}$ Advances in the management of common conditions, such as using antibiotics to treat hypergastrinaemia for bacterial colonisation in peptic ulcer disease, ${ }^{5}$ or the application of topical techniques that non-specialists have read of but not seen used, such as the molecular analysis of human genes ${ }^{6}$ also make good reports. It helps if there are good radiological and pathological features. I have tried to select cases that are appropriate for a general clinical readership as more esoteric cases are better suited to specialist or basic science journals. When uncertain about whether a case is suitable for publication I have sent a synopsis to the journal and left the final decision to editorial staff; this has prevented would be contributors spending time and effort on a case that may not be publishable.

\section{Writing the report}

The headings of introduction, clinical history, comment, and discussion have been used throughout the series. The introduction, clinical history, and $N$ comment are written by the team presenting the case, who take responsibility for its accuracy. The introduction should state clearly the reason why the case is being reported. What are the interesting points? Why should the reader read on? The clinical history should summarise the salient clinical points giving relevant information about presentation, physical signs, and abnormal and relevant normal results of investigations. Space does not allow the case to be written up as a whodunnit in the style of the New England Fournal of Medicine and it was not the intention to try to emulate this series. The usual guidelines about good medical writing apply: short words, short sentences, and no abbreviations. The account preferably should be written on a word processor as this helps subsequent editing even though the journal does not accept the final account on disc. The floppy disc can, if needed, be borrowed by other participants, who may wish to add $\rightleftharpoons$ references or to change text. Spell checking (and virus detecting) software is useful to scan the various contributions. 